\title{
Análisis de errores y producción escrita de español lengua extranjera
}

\author{
Lívia M. T. Rádis BAPTISTA \\ Mackenzie/FEUSP/Unicamp
}

Palavras-chave: língua espanhola - ensino - produção escrita - erros - fossilização

Resumo: $\mathrm{Na}$ primeira parte deste trabalho, apresentamos alguns conceitos básicos dos seguintes modelos: Análise contrastiva, Análise de erros e Interlíngua. Posteriormente, exporemos o resultado de nossa investigação acerca dos erros produzidos por aprendizes universitários de língua espanhola em suas produções escritas.

\begin{abstract}
In the first part of this paper we present some basic concepts concerning to the following models: Contrastive analysis, Error analysis and Interlanguage. Afterward, we expose the results of our investigation on the undergraduate students' errors of Spanish language course in writing composition.
\end{abstract}

Resumen: En la primera parte de este trabajo presentamos algunos conceptos básicos acerca de los siguientes modelos: Análisis contrastivo, Análisis de errores e Interlengua. Enseguida, expondremos el resultado de nuestra investigación de los errores presentes en la producción escrita de estudiantes universitarios de lengua española.

\section{Introducción}

Este trabajo propone demostrar la importancia de una reflexión acerca de los errores para alumnos y profesores. En la primera parte de 
este estudio presentamos algunas consideraciones breves acerca del análisis contrastivo, análisis de errores e interlengua. Luego presentamos el resultado de una investigación llevada a cabo en nuestra universidad a lo largo del primer semestre del año 2002. Seleccionamos cinco producciones escritas como muestra de análisis, teniendo en cuenta los siguientes aspectos: a) la tipología de los errores y b) su frecuencia. La hipótesis inicial era la de que la mayor dificultad sería a nivel de la competencia lingüística, es decir, que los errores predominantes serían del tipo gramatical. Sin embargo, en las producciones del grupo seleccionado, notamos que los errores más frecuentes no eran de ese tipo, sino errores de acentuación y ortografía. Nuestro objetivo, aquí, es el de analizar, identificar e interpretar las posibles causas de los errores presentes en las producciones escritas de los aprendices. Y, de ahí, reformular nuestras clases de producción escrita en español y para ello proponer actividades didácticas con las cuales logremos subsanar dichos puntos de dificultad.

\section{Conceptos básicos}

\subsection{Lingüística Contrastiva}

Conforme Santos Gargallo (1993, p. 27), el término Análisis contrastivo es de Trager (1949). Al principio, el objetivo del Análisis contrastivo era la elaboración de una gramática contrastiva que pudiera aunar, de forma única, las gramáticas descriptivas de dos lenguas y, simultáneamente, pudiera permitir, con alguna exactitud, definir cuáles serían las partes de la estructura que podrían representar dificultades para el aprendiz y cuál sería la naturaleza de tales dificultades en el proceso de aprendizaje. De este modo, presuponía una comparación entre lenguas, lo que no consistía en sí misma el objeto de la Lingüística Aplicada, sino un procedimiento para aclarar los mecanismos subyacentes al proceso de aprendizaje de una segunda lengua. 
Gallisson y Coste (1983, p. 162) señalan que la Lingüística Contrastiva (o diferencial) es una rama de la Lingüística dicha Aplicada y que se ha desarrollado, principalmente, en los Estados Unidos a partir de los años 50. Su preocupación inicial era la comparación entre cada término de dos lenguas, en todos los niveles (fonológico, morfo-sintáctico y, eventualmente, semántico). Posteriormente, a partir de las diferencias entre las dos lenguas proponer métodos apropiados para ser aplicados a la enseñanza de una determinada lengua extranjera. Considerándose las comparaciones sistemáticas, juntamente con el examen de los errores efectivamente cometidos, sería posible explicarse no solamente la naturaleza de esos errores, sino también decidir en cuanto a los medios para su prevención o para su eliminación.

La Lingüística Contrastiva puede asumir dos modalidades: la teórica y la práctica. La modalidad teórica trata de las diferencias y las semejanzas entre dos o más lenguas y su objetivo es el de ofrecer un modelo adecuado para establecer la comparación y determinar cuáles elementos son los comparables. Opera con el concepto de universales lingüisticos, pues interesa cómo una categoría universal puede ser representada en las lenguas comparadas. Ya la modalidad práctica estudia cómo una categoría universal $\mathrm{X}$ se realiza en la lengua A como Y y en la lengua B como diferente. Dicha característica sería la base de las dificultades en el aprendizaje de una segunda lengua. Siendo así, el análisis de las divergencias podría facilitar no sólo la comprensión del proceso de aprendizaje del estudiante sino también facilitar la actuación del profesor. Cabe recordar, aquí, que los universales lingüísticos son los conceptos o rasgos comunes a todas las lenguas naturales existentes. El estudio de los conceptos o rasgos comunes de las lenguas naturales adquirió gran importancia con las investigaciones sobre traducción automática y sobre las gramáticas generativas, que postulan la existencia de similitudes entre las diferentes lenguas. Los trabajos de Lingüística Contrastiva se ocupan, de modo general, de dos lenguas simultáneamente y su presupuesto es la existencia de los universales lingüísticos, mas aún, de la existencia de semejanzas entre las lenguas. 
Para Santos Gargallo (1993) la Lingüística Contrastiva ha desarrollado una investigación seria y fecunda entorno al aprendizaje de segunda lengua ${ }^{1}$ y prueba de ello son los modelos de análisis que actualmente han estimulado muchas investigaciones y trabajos. Los modelos que resultan de las contribuciones de la Lingüística Contrastiva son los siguientes: el de análisis contrastivo, el de análisis de errores y el de interlengua.

\subsection{El Modelo de Análisis Contrastivo (AC)}

Según Santos Gargallo (1993, p. 34-35), este modelo considera la comparación del sistema de la lengua nativa (L1) - del estudiante - con el sistema de la segunda lengua que él está aprendiendo. La comparación comprende los niveles: fonológico, gramatical, léxico y cultural. En cuanto a su metodología, podemos reconocer los siguientes procedimientos:

1. Descripción estructural de L1 y L2;

2. Comparación de las descripciones;

3. Elaboración de una lista de estructuras no equivalentes;

4. Reagrupamiento de las mismas estableciendo una jerarquía de dificultades;

5. Predicción y e descripción de las dificultades;

6. Preparación de materiales de instrucción.

Además de lo antedicho, algunos conceptos fundamentales del modelo son los siguientes:

\footnotetext{
${ }^{1}$ Santos Gargallo (1999, p. 21) señala la diferencia entre Lengua Segunda (L2) y Lengua Extranjera (LE) en los siguientes términos: la L2 es "aquella que cumple una función social e institucional en la comunidad lingüística en que se aprende" ya la LE es "aquella que se aprende en un contexto en el que carecer de función social e institucional”.
} 
1. Concepto de distancia interlingüística: es la distancia que media entre a lengua nativa (L1) y la lengua meta (L2).

2. Concepto de interferencia: cuando un individuo utiliza en una lengua meta (L2) un rasgo fonético, morfológico, sintáctico o léxico característico de su lengua nativa (L1).

3. Error: es un desvío de la norma de la lengua meta, debido a interferencia de la estructura con relación a la lengua nativa.

\subsection{El Modelo de Análisis de Errores (AE)}

El modelo de Análisis de errores (AE) surge al final de los años 60, dentro de la teoría de aprendizaje de segunda lengua y es un puente entre el Análisis contrastivo y los futuros estudios de interlengua. Se inspira en la teoría generativa de N. Chomsky y en las teorías cognitivas y mentalistas de aprendizaje. P. Corder (1967) al llamar la atención hacia lo que denominó dialecto idiosincrático, la lengua de los aprendices, dio impulso a una nueva postura frente al error y a su estudio sistemático. El dialecto idiosincrático se define por sus propias peculiaridades, diferentes de las de las lenguas materna y de las de la lengua meta, conforme nota Barallo (1999, p. 37).

Considerándose lo antedicho, el error pasa a ser entendido como si fuera un desvío sistemático y recurrente que afectaría la norma estándar de la lengua meta. Sería positivo e inevitable. Además, el error sería una prueba de que el proceso de aprendizaje se estaría produciendo.

En la enseñanza comunicativa de lenguas esa nueva postura frente al error va a ser muy importante. En cuanto a su aplicación a la enseñanza de lenguas, conforme Vázquez (1999, p. 25) el análisis de errores es fundamental para profundizar un conocimiento acerca del proceso de aprendizaje y, en consecuencia de ello, para definir pautas para la enseñanza y la actuación e intervención del profesor. Por lo tanto, el análisis de errores puede ofrecer información lingüística sobre los problemas gramaticales todavía no analizados. Además de eso, permite que el profesor desarrolle criterios para lo qué corregir, como y cuándo hacerlo. 
Según Baralo (1999, p. 38), son las siguientes etapas del modelo:

1. Reconocimiento de la idiosincrasia de esa lengua del aprendiz.

2. Descripción: se basa en la comparación del dialecto idiosincrático del alumno con su lengua materna y con la lengua objeto, mediante el mismo modelo formal de análisis del conjunto de categorías lingüísticas y sus relaciones sintácticas, comunes a los dos sistemas contrastados.

Para Santos Gargallo (1993, p. 85) la sistematización del Análisis de errores consiste en:

1. Determinación de los objetivos (globales o parciales)

2. Descripción del perfil del informante.

3. Selección del tipo de pruebas (test, redacción, traducción, etc.).

4. Elaboración de la prueba.

5. Realización de la prueba (grupo o grupos de estudiantes).

6. Identificación de los errores.

7. Clasificación de los errores detectados de acuerdo con una taxonomía gramatical basada en la gramática estructural.

8. Determinación estadística de la recurrencia de los errores.

9. Descripción de los errores con relación a las causas o causa.

10. Identificación de áreas de dificultad, según una jerarquía.

11. Programación de una terapia para el tratamiento de los errores.

12. Determinación del grado de irritabilidad causado en el oyente.

\subsection{La noción de interlengua (IL)}

Conforme Fernández (1997, p. 20), las descripciones de la interlengua se inician con el Análisis de Errores y, enseguida, al considerar que todo formaba parte de la lengua del aprendiz empiezan a tener en cuenta no 
sólo los errores. El Análisis de errores nace con una finalidad didáctica y, luego, se asocia a modelos lingüísticos, psicolingüísticos y sociolingüísticos. Además de eso, incorpora la interacción comunicativa y la dimensión pragmática de la lengua.

La interlengua (IL) es una etapa obligatoria en el aprendizaje y es un "sistema lingüístico interiorizado", que puede evolucionar y convertirse, en cada vez más complejo, y sobre lo cual el aprendiz tiene intuiciones. Por lo tanto, podemos considerar que (Fernández, 1997 apud Frauenfelder et al., 1980):

[...] la IL (interlengua) constituye una etapa obligatoria en el aprendizaje y se definiría como un 'sistema lingüístico interiorizado, que evoluciona tornándose cada vez más complejo, y sobre el cual el aprendiz posee intuiciones. [...] Las investigaciones en IL demuestran que, en cada estadio, las producciones de los aprendices obedecen a unos mecanismos e hipótesis sistemáticas. [...] Con todo, esta sistematicidad es variable, tanto porque las hipótesis se van reestructurando progresivamente, para dar paso a otra etapa- variabilidad sistemática -, en la línea de Labov, como por las fluctuaciones propias de cada etapa- variación libre - [...]

\section{Clasificación de los errores}

En cuanto a la clasificación de los errores, Santos Gargallo (1993,p. 91-96) propone algunos criterios para su clasificación que pueden orientar una investigación basada en el modelo de Análisis de errores: a) criterio descriptivo; b) criterio pedagógico; c) criterio etiológico-lingüístico; d) criterio gramatical y e) criterio comunicativo. A continuación, explicaremos, sumariamente, en que consisten esos criterios.

El criterio descriptivo se basa en la forma en la que la estructura superficial de las instancias se presenta alterada. Puede asumir las siguientes formas: a) omisión; b) adición - comprende 1.doble marca, 2. regularización y 3. simple adicción; c) formación errónea y d) ausencia de orden oracional.

SIGNUM: Estud. Ling., Londrina, n. 5, p. 49-75, dez. 2002 
El criterio pedagógico se basa en la distinción de Chomsky entre competencia y actuación/desempeño. Siendo así, los errores pueden ser sistemáticos o transitorios. Además, hay errores fosilizables y errores fosilizados, errores inducidos y errores creativos, errores individuales y errores colectivos, errores de producción escrita y errores de producción oral, nota Barallo (1999, p. 40).

Según el criterio etiológico-lingüístico, basado en el concepto de transferencia lingüística, hay errores interlingüísticos y/o errores intralingüísticos. Para Barallo (1999, p. 40), todavía hay los errores de simplificación.

El criterio gramatical se basa en la categoría gramatical afectada por el error e incluye todos los niveles de la gramática tradicional (morfología, sintaxis, semántica y léxico). Ese tipo de taxonomía es muy frecuente y su objetivo es el de medir la competencia gramatical del estudiante. Pueden ser: errores fonológicos, ortográficos, morfológicos, sintácticos, léxicos, semánticos y pragmáticos.

El último criterio - el comunicativo - se basa en el efecto comunicativo desde la perspectiva del oyente, pues se evalúan los errores como obstáculos para la transmisión del mensaje. Pueden ser locales cuando afectan elementos individuales de la oración y globales cuando se refieren a toda la oración y provocan ruptura en la comunicación. Para Barallo (1999, p. 40) hay errores de ambigüedad, errores irritantes y errores estigmatizantes.

\section{Análisis de errores en la producción escrita de aprendices brasileños de español}

Empleamos, en este trabajo, aunque parcialmente ${ }^{2}$, la propuesta de

\footnotetext{
${ }^{2}$ Un trabajo más profundo exigiría una comparación entre las producciones de diferentes grupos, cruce de datos, además de un análisis de producciones por más tiempo. Nuestra propuesta es la de un ejercicio de análisis que comprueba la vitalidad del modelo.
} 
sistematización del procedimiento en Análisis de Errores recogida de Santos Gargallo (1993, p. 85). Adoptamos los siguientes procedimientos:

- Determinación de los objetivos (globales o parciales).

- Descripción del perfil del informante.

- Selección del tipo de pruebas (test, redacción, traducción, etc.)

- Elaboración de la prueba.

- Realización de la prueba (grupo o grupos de estudiantes).

- Identificación de los errores.

- Clasificación de los errores detectados de acuerdo con una taxonomía gramatical basada en la gramática estructural.

- Descripción de los errores con relación a las causas o causa.

\subsection{Determinación de los objetivos (globales o parciales)}

Nuestros objetivos son los siguientes: a) identificar e interpretar las causas de los errores en la producción escrita de aprendices de español como lengua extranjera; b) a partir del conocimiento de las causas de estos errores reorganizar el curso de producción escrita, seleccionar materiales y contenidos e intervenir en la corrección; c) medir la competencia lingüística de los aprendices pues suponíamos que esa sería fundamental para su producción escrita.

\subsection{Descripción del perfil del informante.}

Son alumnos de la Carrera de Letras (Portugués/Español) de una universidad privada de la capital de São Paulo. La Universidad está ubicada en el centro de São Paulo y posee tradición en la enseñanza superior, especialmente, en las carreras de Derecho y de Educación. Son alumnos del sexto semestre, lo que corresponde al último semestre del tercer curso y que han tenido por lo menos dos años y medio de enseñanza del idioma. 
El curso tienen duración de cuatro años y se imparte en semestres. Los alumnos tienen clases de lengua española desde el primer curso. A lo largo del primer año, tienen cuatro horas de clase por semana. A partir del segundo curso, además de literatura española e hispanoamericana, tienen cuatro horas semanales de clase de lengua y dos horas de producción escrita, estructura que se mantiene hasta el último semestre. Además de eso, la lengua nativa es el portugués, en su variante brasileña, aunque haya un informante que es portugués, de distinta variante. En la universidad hay otra opción de carrera que es la de Inglés-Español.

\subsection{Selección del tipo de pruebas (test, redacción, traducción, etc.)}

Seleccionamos muestras de la producción escrita de los aprendices del curso de producción y revisión de textos. En el sexto semestre, trabajamos el discurso argumentativo, por lo tanto, los textos recogidos son expositivos-argumentativos.

\subsection{Elaboración de la prueba}

Para estimular la producción escrita, el profesor hizo un trabajo previo de comprensión lectora, a partir de un texto de Antonio Gala, "Los medios de incomunicación", seleccionado del periódico español del El País, del año 1997. Posteriormente, trató del tema de la globalización y los medios de comunicación. Los aprendices tuvieron que redactar un texto en el que presentasen su punto de vista acerca de las relaciones entre la globalización y la felicidad. En el cuerpo de este trabajo presentamos los textos producidos pasados a ordenador y sin ninguna identificación del aprendiz con la finalidad de preservarle su identidad. Cabe agregar que respetamos el modo de presentación de los originales e intentamos conservar todas las características del texto original. Solamente el primer texto no fue manuscrito, sino pasado a ordenador por el propio aprendiz. 


\subsection{Realización de la prueba (grupo o grupos de estudiantes)}

Conforme ya mencionamos, son alumnos universitarios de la carrera de Letras. Las producciones fueron individuales y pudieran utilizar materiales de consulta.

\subsection{Identificación de los errores}

A continuación presentamos las producciones de los aprendices y señalamos algunos de los errores analizados. En este proceso, nos interesó enfatizar los errores teniendo en cuenta el criterio gramatical.

3.7. Clasificación de los errores detectados de acuerdo con una taxonomía gramatical basada en la gramática estructural.

Teniendo en cuenta la opción por el criterio gramatical, aunque, sin duda reconozcamos que para la producción oral y escrita los errores discursivos apuntados por Sonsoles Fernández (1999) sean de extremada importancia, centraremos nuestro análisis en las categorías afectadas por el error en los niveles de la gramática. Haremos hincapié en los errores gramaticales, considerándose en nuestro análisis las propuestas de Barbieri Durão (1999) y de Sonsoles Fernández (1997), además de la Santos Gargallo (1997).

\section{8. Descripción de los errores con relación a las causas o causa}

A partir de las muestras seleccionadas proponemos posibles explicaciones para los errores observados, pues nos parece de gran importancia identificar e interpretar las causas de estos errores para planificar las clases, corregir y evaluar las producciones futuras de los aprendices.

SIGNUM: Estud. Ling., Londrina, n. 5, p. 49-75, dez. 2002 


\section{Análisis de las cinco producciones escritas de los aprendices}

\section{TEXTO 1}

\section{Globalización - Una falsidad impuesta}

Si 1 os gobernantes de los países más ricos dicen que la globalización és buena, está claro que la globalización no lo és para los países en la miseria pues después de anos de globalización aun dos tercios de la población mundial sigue siendo mucho pobre.

La ideologia de la globalización es que todos dependamos de todos. Se sugiere un equilibrio en la dependencia mutua, un esfuerzo y un beneficio compartidos, un sueño estupendo. Un sueño aceptado por todos los países del mundo para lograr el bien común.

Pero el sueño és falso todavia. La dependencia es profunda y perversa. Los más ricos necesitan explotar a los que son más pobres para poder prosperar y los más pobres tienen que dejarse explotar para poder sobrevivir en suyas miserables condiciones.

Por encima de todo, la globalización es una perversión económica. La información global, la cultura global, no existen. Eso se encuentra en manos de las grandes empresas, que sólo quieren saber de lucros y, por eso, han convertido los gobiernos del mundo desarrollado en sus suditos.

La economía global ha roto todas las fronteras, como por exemplo las do tipo moral, y se mueve en un territorio donde, por supuesto, rige la ley del más fuerte. Además los más fuertes no aniquilan por completo a los más fracos. A estos se les dan lo justo para que sobrevivan al límite de sus fuerzas y, así, seguiren esclavos.

Por eso la globalización permite algunos buenos gestos, como el perdón parcial o selectivo de la deuda a los países pobres. Una forma de mantenerlos vivos y rentables.

Dicen sus seguidores que la globalización es buena para todos, que sólo hay que corregir sus efectos malos. Es como decir que la esclavitud es buena para el amo y para el esclavo, que sólo hay que inventar la forma de cambiar sus efectos perversos. 


\section{Comentario de los errores y descripción de los errores}

Observamos, básicamente, tres errores gramaticales: 1) uso de la forma plena ("suyas") y no apocopada ("sus") ${ }^{3}$, como se nota en el ejemplo: "en suyas miserables condiciones"; 2) ausencia de la contracción preposición (de) con artículo masculino singular (el), en "las do tipo" y 3) uso de infinitivo flexionado " $\mathrm{y}$, así, seguiren esclavos", estructura que no existe en español.

Además de los errores gramaticales señalados, notamos algunos ortográficos y de acentuación que, aunque no sean nuestro foco de análisis, decidimos incluirlos aquí. Vale aclarar que siempre que entendamos que sea pertinente nos refererimos a otros tipos de errores. Sin embargo, insistimos, en el momento nos ocupamos de los errores gramaticales. Son ejemplos de errores de acentuación: "és” (monosílabo no acentuado), "ideología" (hiato acentuado) y de errores de ortografía: "exemplo" (uso de "x" por "j"), fraco (uso de "r" por "l", además de la inadecuación contextual y semántica). Hay un caso de error ortográfico y de acentuación que es el de "suditos" (por súbditos"). Además de la separación del sujeto por medio de comas en "La información global, la cultura global, no existen". Hay otro problema en el texto que es referencial, presente en "Eso se encuentra en manos" que debería enlazar, lógicamente, con el antecedente "la información global" y "la cultura global" y no negarlo. Otro error es el uso de "todavía", forma de adverbio de tiempo semejante a aún. Cuando le preguntamos al informante el motivo de ese uso, nos explicó que había pensado en el sentido de su uso en portugués. Es interesante señalar que el informante posee la variante portuguesa peninsular y se utiliza a menudo de esta construcción.

\footnotetext{
${ }^{3}$ Término utilizado por Gómez Torrego (1998, p. 76).
} 


\section{Explicación e interpretación de los errores}

En el caso del alumno anterior, notamos que sus errores son de ortografía y de acentuación. Hay otros relacionados con la organización textual, pero no afectan la cohesión y la coherencia del texto. Sin embargo, llama la atención que los errores observados son frecuentes, en general, en los niveles básicos y no propios del nivel de interlengua en el que está el aprendiz. Probablemente eso se explique por algún problema relacionado con la cantidad de input o entonces con relación a la corrección. En cuanto a los errores gramaticales notamos que el primer error señalado, la falta de contracción de la preposición y del artículo, no debería ocurrir en este nivel. Hay que señalar que el aprendiz utiliza correctamente los artículos e, incluso, una contracción correcta en "rige la ley del más fuerte". Otro error gramatical identificado es la forma plena del posesivo en lugar de la apocopada. Hay que recordar que no se utiliza la forma plena delante de sustantivos. La elección de una u otra forma depende de la posición y de la función del posesivo en la frase. Usamos la forma apocopada delante de sustantivos y las plenas cuando los sustituimos. En contrapartida, en portugués no existe esa diferencia de formas. Una de las causas de ese error es la no sistematización de las formas y del uso del posesivo. Conviene agregar que para Barbieri Durão (1999, p. 127-128), al emplear la forma plena en lugar de la apocopada, el aprendiz comete un error en el uso de la apócope y según esa autora:

En lo que atañe al fenómeno de la apócope, los lusohablantes cometen desviaciones de la al parecer norma porque en la lengua portuguesa hay una única posibilidad para formas antepuestas y pospuestas al nombre, que corresponde a dos posibilidades en la lengua española: una para la anteposición y otra para la posposición. De ahí que los aprendices puedan emplear tanto apócope en situaciones en las que no se suele emplear, y pueden no emplearla cuando se requiere. [...] 
Otro error observado fue el empleo del infinitivo flexionado. Recordamos que en portugués existen dos posibilidades: el infinitivo personal (por lo tanto lo podemos flexionar) y el infinitivo no personal (no flexionado). En ese sentido, hay algunas posibilidades de uso para una y otra forma. Según Celso Cunha e Lindley Cintra (2001,p. 485), “el empleo de las formas flexionada y no flexionada del infinitivo es una de las cuestiones más controvertidas de la sintaxis portuguesa" y los autores prefieren tratar el asunto en términos de tendencias de uso y no de reglas. Conforme Barbieri Durão (1999, p. 124) se trata de una interferencia peculiar a los lusohablantes, pues la posibilidad de flexionar el infinitivo en el morfema de persona y número es una característica de la lengua portuguesa.

\section{TEXTO 2}

\section{¿Cuál es la relación entre la globalización y la felicidad?}

Pienso que la globalización por un lado es buena en el sentido que por medio de la informática no exista más distancia dentro del mundo, pero ella cambia poco a poco los valores culturales, transformando las personas cada vez más individuales y consumistas y estas personas se olvidan que la felicidad no estas en el avanzo tecnológica, en los bienes materiales y sí ellá está en una sonrisa de una niña que tiene amor, armonía en su casa, en su familia, esta en el canto de los pajaros que quasi no se oye más.

La felicidad esta en la solidariedad con el otro, el respeto y finalmente en la amistad que no si ve más, pues con el avanzo las personas no tienem tiempo de vivir la vida y aprovechar las personas que aman el medio ambiente que hay, muy poco en las grandes ciudades.

SIGNUM: Estud. Ling., Londrina, n. 5, p. 49-75, dez. 2002 


\section{Comentario de los errores y descripción de los errores}

Además de los errores gramaticales del recuadro, hay errores ortográficos y de acentuación en la producción del informante y de ahí son ejemplos de errores del primer tipo: "quasi" (por "casi"), "avanzo" (por "avance") y "tienem" (por "tener") y del segundo tipo: "ellá" (por “ella"), "pajaros" (por "pájaros") y "esta” (por "está", verbo). Notamos aún en el primer párrafo la falta de la correlación entre las ideas pues menciona "Pienso que la globalización por un lado es buena" y no señala la oposición de esa idea a través de "por otro lado". Entre los errores gramaticales, notamos errores de dos tipos, básicamente: de concordancia en género y en número gramatical y de uso incorrecto del demostrativo. En cuanto al primer tipo, el aprendiz empleó la forma femenina para calificar al sustantivo "avance" (concordancia en género) y en el caso de concordancia en persona empleó la segunda persona del verbo en lugar de la tercera ("la felicidad no estas"). En cuanto al empleo del demostrativo, utiliza "estas personas" por "esas personas", pues ya se había referido a las personas en su texto.

\section{Explicación e interpretación de los errores}

Podemos observar que los errores del aprendiz se deben probablemente a una mejor sistematización de la ortografía y de las reglas de acentuación del español. En cuanto al caso de "el avanzo tecnológica", sabemos que el adjetivo acompaña al sustantivo en género (masculino o femenino) o en número (singular o plural). En nuestro ejemplo eso no ha ocurrido. Nos parece que su fallo se debe a la desatención y no al conocimiento de dicha regla, pues en su texto emplea correctamente la concordancia entre "globalización" y "buena". Ya el error de concordancia, cuyo ejemplo es "la felicidad no estas", puede indicarnos que no ha habido una sistematización del paradigma verbal y se trata de un error frecuente 
en los niveles iniciales que tiende a fosilizarse. Además de esos ejemplos, el uso del demostrativo se asocia a un problema a nivel discursivo.

\section{TEXTO 3}

\section{Los médios de Incomunicación}

Parece que la liberdad de expresión en algunos países, después de la dictadura, volvió la gente tonta.

Se puede notar eso a través de los médios de comunicación, que están cada vez peor: la televisión, el cine, los periódicos y revistas, músicas etc.

En Brasil cuando había el regimen dictarial, los compositores tenían que estudiar estilística para escribir sus canciones de manera que los políticos no comprendesen y el pueblo si. Eso hizo con que estas obras volviesen grandes poemas y muchas son estudiadas por literarios. Hoy tenemos pocos artistas que trabajan su talento como ellos. La televisión volvió una gran basura. Los programas enseñan mujeres desnudas, novelas de poco contenido o mejicanas o promoven los peores cantantes y bandas.

El cine produce películas violentas (Arnold Schwazeneger etc), pocas son las que pasan una buen mensagen.

Los periódicos, además de mal escribidos, se preocupan a enseñar la vida de los famosos.

Como se observa los médios de comunicación se volveran incomunicable, buen era el tiempo en que se tenía que pensar para comunicar.

\section{Comentario de los errores y descripción de los errores}

Notamos errores de diferentes tipos en la producción del aprendiz. Entre los errores ortográficos señalamos los siguientes: liberdad (por 
"libertad"), "dictarial" (por "dictatorial") "mensagen" (por "mensaje”). Hay errores de acentuación: "médios regimen si", cuyas las formas correctas serían respectivamente: “medios, régimen, sî". Presenta también error lexical: "compositores" (por "cantautores"). Empleo incorrecto de la forma apocopada en "buen era el tiempo" por "bueno era el tiempo". Entre los errores gramaticales, observamos los siguientes:

1. Error en la forma verbal del pretérito imperfecto de subjuntivo y omisión del pronombre complemento en función de objeto directo (acusativo): "los políticos no comprendesen" por "los políticos no (las) comprendiesen".

2. Error en la forma verbal del pretérito (indefinido o pretérito perfecto) y error de concordancia en número: "médios de comunicación se volveran incomunicable" por "medios de comunicación se volvieron/se han vuelto incomunicables".

3. Error en la forma del presente de indicativo del verbo diptongable "promover": "promoven los peores cantantes y bandas" por "promueven los peores cantantes y bandas".

4. Error en la concordancia sustantivo y adjetivo: "médios de comunicación, que están cada vez peor", por "los medios de comunicación, que están cada vez peores, [...]”.

5. Error en el empleo de la forma del participio irregular en "los periódicos, además de mal escribidos" por "los periódicos además de mal escritos".

6. Error en el empleo de la preposición que acompaña al verbo en "se preocupan a enseñar" por "se preocupan por enseñar".

\section{Explicación e interpretación de los errores}

Observamos que el aprendiz presenta un número elevado de errores gramaticales. Uno de estos errores es el empleo de formas que, 
obligatoriamente, son diptongables en español (ejemplos a,b y c). La diptongación consiste en una alteración de la raíz cuando lleva el acento tónico. En muchos casos, la vocal se transforma en diptongo por el peso del acento. Las vocales se diptongan del siguiente modo: $-\mathrm{e}=-\mathrm{ie} ;-\mathrm{o}=-\mathrm{ue}$, $-\mathrm{i}=\mathrm{ie}$. Este fenómeno no ocurre en portugués y debido a eso hay dificultad para la asimilación de las formas diptongables.

Otro error que llama la atención es la omisión del pronombre complemento (ejemplo a) que sería obligatorio antes de la forma verbal. En portugués se suele omitir la forma pronominal en dichos casos, especialmente, en el habla coloquial y en la modalidad del portugués brasileño.

El informante presenta aún error en cuanto al empleo de la forma del participio. En el sistema del español hay verbos que tienen los dos tipos de participio; la forma irregular es la que actúa solamente como adjetivo y jamás como verbo, excepto en los casos de los siguientes verbos: freír, proveer e imprimir, pues en dichos verbos los participios regulares e irregulares pueden actuar también como verbos, conforme Gómez Torrego (1998, p. 140). El verbo escribir, a su vez, presenta una forma irregular (escrito/a), siendo que este verbo en lugar del formante - ido (de la mayoría de los casos) presenta el formante -to, según Seco (1994, p. 289). Probablemente el aprendiz aplicó una regla equivocada para el participio, o sea, no está claro todavía el uso de la forma irregular.

Otro error es el empleo de la preposición incorrecta (ejemplo f). Barbieri Durão (1999, p. 131), al tratar de los usos incorrectos de preposiciones, señala que el uso de las preposiciones:

[...] es determinado por las relaciones sintagmáticas de un enunciado y esas pueden ser divergentes de una lengua para otra. Cuando coinciden suele haber transferencia positiva, pero cuando son contrastivas, normalmente hay transferencia negativa o interferencia.

SIGNUM: Estud. Ling., Londrina, n. 5, p. 49-75, dez. 2002 
En nuestro ejemplo, notamos que hay un desconocimiento, por parte del aprendiz, del régimen del verbo "preocuparse" que, en español, admite las siguientes posibilidades: preocuparse con, de, por algo (Bescherelle, 1984, p. 235).

\section{TEXTO 4}

\section{MEDIOS DE INCOMUNICACIÓN}

La tecnología ha producido un mundo cada vez más interconectado, es decir muchas personas hoy puedem comunicarse de un país a otro sin saliren de sus casas, solo con un toque en la computadora.

Para algunas personas ese fato puede ser visto como una característica de la globalización así que hoy dia todos puedem se comunicar com mucha facilidade.

Pero, decir que el mundo está, realmente, globalizado no es una buena cosa pues, mesmo que las personas puedam comunicarse con todo el mundo en minutos no podemos decir que todos tienen las mismas oportunidass (i) lo que seria cierto dentro del concepto de globalización.

Mismo que exista el livre mercado entre grupos de países, los países "ricos! Continuam buscando en los países "pobres" productos e mano de obra más baratas para el trabajo en sus empresas.

Con eso podemos decir que la globalización solo existe encuanto ideal y no en la realidad.

\section{Comentario de los errores y descripción de los errores}

Observamos los siguientes errores ortográficos: "puedem" (por “pueden”), "puedem” (por “pueden") “com” (por “con”) y “continuam" 
(por "continúan") en estos casos el aprendiz ha empleado la m final en los términos. Según Barbieri Durão (1999, p. 105), en portugués la -n final pasó a -m final, hecho que se dio en el curso del siglo XIII y son raros vocablos que terminen en - $\mathrm{n}$ y, por ello, el aprendiz lusohablante de español utiliza la regla de su lengua. Otros errores ortográficos que hemos notado: "facilidade" (por "facilidad"), "oportunidas" (también error de formación del plural), "livre" (por "libre"). En cuanto a los errores de acentuación, hay los siguientes: "dia" (por "día"), "seria" (por "sería") y "solo" (por "sólo"). El aprendiz utiliza también la forma "solo existe encuanto ideal" por "sólo existe en cuanto / como ideal". Hay un error en relación a la forma y, en consecuencia, el sentido es otro. Recordamos que la forma en cuanto tiene los siguientes usos en español: 1) inmediatamente; 2) como. Sin embargo, con la acepción del portugués hay que emplear la forma "mientras".

Entre los errores gramaticales, señalamos los siguientes:

1. Uso del infinitivo flexionado: "sin saliren de sus casas".

2. Colocación pronominal errónea: "puedem se comunicar" (por "pueden comunicarse").

3. Error en la expresión de la concesión: "mesmo que las personas puedam comunicarse" (por "Aunque las personas puedan comunicarse") y "Mismo que exista el livre mercado" (aunque exista el libre mercado").

\section{Explicación e interpretación de los errores}

El aprendiz, igual que el del primer texto, emplea el infinitivo flexionado (ejemplo a). Hemos observado que este tipo de error es común a los lusohablantes lo que puede indicar una interferencia con el portugués. Ya en cuanto al ejemplo b, hay error de colocación pronominal. Según Barbieri Durão (1999,p. 125) hay interferencia en estos casos pues los 
aprendices tienden a posicionar las formas pronominales conforme lo habitual en portugués. El uso de "mesmo" por "aunque" es un error frecuente en aprendices lusohablantes del español. Recordamos que en español "mismo" es un adjetivo que se aplica a un nombre para expresar que aquello que designa es una única cosa: Los dos niños llevan el mismo apellido. Esta chica y la (chica) que vimos ayer son la misma (chica). Si utilizamos con el artículo neutro, significa "la misma cosa": Me hablas lo mismo siempre. También puede ser sinónimo de igual, muy semejante o de la misma clase: Vestimos siempre del mismo color. Todavía hay la expresión enfática: Se la entregó a él mismo. Existe el uso de mismo como adverbio: Vimos los hechos desde mi piso mismo. En el caso que nos interesa, el uso del portugués de "mesmo que" es de conjunción subordinativa concesiva y es este valor que el aprendiz desea obtener y por ello utiliza la forma del portugués en español.

\section{TEXTO 5}

\section{Medios de Comunicación}

Atualmente se habla mucho de los medios de comunicación en todo el mundo.

Existe varios medios para los hablantes se comunicaren, como por ejemplo: pelo radio, la tele, la internet con el correo electrónico (email), el teléfono y la prensa.

Con la globalización el mundo se encuentra mas "próximo", pues para hablar con familiares y amigos que se encuentran en otro país basta acesar la internet, pero esta cultura del e-mail corroe todos los pilares de la gramática, la sintaxis y la ortografía criando así su propria lenguaje. 
Para las empresas un mundo globalizado representa mejores garantías de acuerdos, negocios y una remuneración cada vez mayor, pero ocurre también un aumento de la tasa de desempleo en diversos países del mundo inclusive el Brasil.

Es muy difícil hablar sobre los medios de comunicación y sobre la globalización, pues son temas que tienen muchos tópicos que hacen con que refletimos un poco con los "prós" y "contras" del progreso del mundo pela tele, pela internet, por el radio y por el teléfono.

Puedo concluir que los medios de comunicación y la globalización son muchos importantes para toda la humanidad.

\section{Comentario de los errores y descripción de los errores}

Observamos algunos errores de ortografía y de acentuación. Son ejemplos del primer tipo de error: "atualmente" (por "actualmente"), "acesar" (por "acceder"), "criando" (por "creando") y "propria" (por "propio" - error de concordancia). Con relación a la acentuación, notamos: "mas próximo" (por "más próximo", tilde diacrítica) y "prós" (por "pros"). Hay también el uso erróneo de la forma "refletimos" por "reflexionamos", un error semántico. Además de eso, hay los errores gramaticales:

1. Concordancia en número: "Existe varios medios" por "Existen varios medios".

2. Concordancia en género: "propria lenguaje" por "propio lenguaje".

3. Colocación de pronombre e infinitivo flexionado: "se comunicaren" por "comunicarse".

4. Uso de artículo delante del nombre propio de país: "el Brasil".

5. Ausencia de contracción de preposición y artículo: "pelo radio" (por "por la radio", error en el uso del artículo) y "pela tele", 
"pela internet" (respectivamente, "por la tele", "por la internet".

6. Uso erróneo de mucho en lugar de muy: "mucho importante" (por "muy importante")

\section{Explicación e interpretación de los errores}

Nos parece que los errores de acentuación y ortográficos se deben a una no sistematización de las formas y no asimilación de algunas reglas básicas, a nivel de acentuación. El error de concordancia se explica, probablemente, por una desatención a la forma correcta (ejemplo b). En cuanto al ejemplo c, puede ser una interferencia con el portugués, puesto que en esa lengua empleamos el género femenino. En cuanto al empleo del infinitivo flexionado es el tercer caso, lo que demuestra la reincidencia de este tipo de error en la producción de lusohablantes. Además de eso, otro error común es el de la colocación pronominal que en portugués es diferente y ocurre en el ejemplo c.

Todavía hace faltar señalar el uso de artículo delante de nombre propio (ejemplo d). Según Barbieri Durão (1999, p. 113), en tales casos hay contagio con la LM, puesto que en portugués es más usual el empleo del artículo determinado con nombres de países. Se trata de una interferencia.

Otro error es la no contracción de la preposición con el artículo determinante (ejemplo e). En portugués disponemos de más posibilidades de este tipo de contracción que en español. En los ejemplos seleccionados, el aprendiz aplica una regla de su lengua materna y eso indica un caso de interferencia.

En fin, el último ejemplo es el empleo de la forma mucho por muy, que sería la correcta. El portugués dispone de una única forma para el adverbio de intensidad y pronombre indefinido que es "muito". La forma invariable corresponde siempre al adverbio y en los casos en los que flexiona acompaña al sustantivo en género y en número. En contrapartida, 
el español presenta dos formas: muy y mucho, variables según su función en la frase. $\mathrm{Al}$ aprendiz lusohablante en los niveles inciales le cuesta identificar y emplear esas formas. Es uno de los errores comunes que pueden venir a fosilizarse.

\section{Propuestas prácticas con vistas a la superación de los errores analizados}

Teniendo en cuenta los problemas identificados en este estudio, decidimos desarrollar a lo largo del semestre algunas tareas facilitadoras que podrán ayudar a la mejor fijación de las formas correctas. Cabe señalar que disponemos de un curso de un semestre para trabajar con dichos aspectos.

De inicio, pretendemos seleccionar materiales y preparar ejercicios con los cuales sea posible trabajar los puntos divergentes en cuanto a la ortografía entre las dos lenguas. Consideramos la posibilidad de una investigación de la propia historia de evolución del idioma y además de eso del uso más frecuente del diccionario como material de consulta. Otra posibilidad de trabajar que tal vez pueda resultar interesante para los aspectos ortográficos es el dictado.

Con relación a la acentuación, hace falta sistematizar mejor las reglas básicas de acentuación en español y proponer más ejercicios que puedan facilitar la memorización, tal vez con apoyo en imágenes y palabras.

Los errores gramaticales van a exigir un trabajo específico. Nos parece posible trabajar de modo contrastivo las dos lenguas y hacer hincapié en los aspectos divergentes sin ignorar las similitudes. Por tratarse de un curso de Letras y de futuros profesionales, nos parece conveniente introducir un trabajo de reflexión lingüística que permita a los aprendices una auto percepción de sus propios errores y una toma de conciencia del papel del error sea para su propio aprendizaje sea para su actuación como profesor de español. 
Considerándose lo antedicho, expusimos algunas propuestas que podremos ejecutar a medio y a largo plazo; de este modo nos pareció extremadamente importante hacer un estudio igual el que aquí hicimos con la finalidad de identificar, reconocer y explicar algunos de los errores más frecuentes del grupo.

\section{Consideraciones finales}

En las producciones escritas analizadas notamos los siguientes aspectos:

1. En general, los aprendices presentan errores frecuentes del tipo ortográfico y de acentuación. Lo antedicho nos puede indicar que hace falta sistematizar, de modo más puntual, esos aspectos del idioma a través de ejercicios y de ejemplos. Algunos de esos errores se deben al desconocimiento de reglas y otros a un fallo en la atención.

2. Los aprendices, en su mayoría, coinciden en cuanto al uso del infinitivo flexionado y la colocación pronominal equivocada. Considerándose los ejemplos, eso se debe a una interferencia con el portugués debido a la diferencia entre esa lengua y el español.

3. Notamos que hay algunos errores semánticos y que se deben a un desconocimiento de la forma adecuada para expresar determinado sentido en español.

4. Además de los errores señalados, muchos aprendices presentan problemas en cuanto a la organización del texto argumentativoexpositivo, pues apenas utilizan estructuras de mayor complejidad del idioma. A pesar de ello, los textos producidos son comprensibles y esos fallos no perjudican su comprensión. 
Considerándose lo antedicho, esperamos reformular nuestro curso de producción escrita en español y lograr mejores resultados en nuestras clases. Hace falta decir que una propuesta de análisis similar a la expuesta constituye una herramienta importantísima para que profesores y alumnos puedan comprender mejor el proceso en el cual están involucrados, o sea, el de enseñanza y aprendizaje del español como lengua extranjera.

\section{BIBLIOGRAFÍA}

BARALO, M. La adquisición del español como lengua extranjera. Madrid: Arco/ Libros, 1999.

BARBIERI DURÃO, A. B. A. Análisis de errores e interlengua de brasileños aprendices de españoly de españoles aprendices de portugués. Londrina: Editora da UEL, 1999.

.; ANDRADE, O. G. Problemas de ensino/ aprendizagem de brasileiros estudantes de espanhol. t. I. Londrina, Paraná: Editora da UEL, 2000.

BESCHERRELLE. El arte de conjugar en español. Diccionario de 12000 verbos. Paris: Hatier, 1984.

CUNHA, C.; CINTRA, L. Nova Gramática do Português Contemporâneo. 3. ed. rev. Nova apresentação. Rio de Janeiro: Nova Fronteira, 2001.

GÓMEZ TORREGO, L. Gramática didáctica de español. Madrid: SM, 1998.

SANTOS GARGALLO, I. Análisis Contrastivo, Análisis de Errores e Interlengua en el marco de la Lingüistica Contrastiva. Madrid: Síntesis, 1993.

SECO, M. Gramática esencial del español. Introducción al estudio de la lengua. Madrid: ESPASA CALPE, 1994.

SONSOLES FERNÁNDEZ. Interlengua y análisis de errores en el aprendizaje del español como lengua extranjera. Madrid: EDELSA: 1997.

SIGNUM: Estud. Ling., Londrina, n. 5, p. 49-75, dez. 2002 75 